\title{
Template-based searches for gravitational waves: efficient lattice covering of flat parameter spaces
}

\author{
Reinhard Prix \\ Max-Planck-Institut für Gravitationsphysik (Albert-Einstein-Institut), Am Mühlenberg 1, \\ 14476 Golm, Germany \\ E-mail: reinhard.prix@aei.mpg.de
}

Received 10 April 2007, in final form 3 July 2007

Published 19 September 2007

Online at stacks.iop.org/CQG/24/S481

\begin{abstract}
The construction of optimal template banks for matched-filtering searches is an example of the sphere covering problem. For parameter spaces with constant-coefficient metrics a (near-) optimal template bank is achieved by the $A_{n}^{*}$ lattice, which is the best lattice covering in dimensions $n \leqslant 5$, and is close to the best covering known for dimensions $n \leqslant 16$. Generally, this provides a substantially more efficient covering than the simpler hyper-cubic lattice. We present an algorithm for generating lattice template banks for constant-coefficient metrics and we illustrate its implementation by generating $A_{n}^{*}$ template banks in $n=2,3,4$ dimensions.
\end{abstract}

PACS numbers: 95.85.Sz, 95.75.Pq, 95.75.-z

\section{Introduction}

The detection of gravitational waves (GWs) in the noisy data of detectors ideally requires the knowledge of the signal waveform, in order to coherently correlate the data with the expected signal by matched filtering. Depending on the type of astrophysical sources considered, however, one typically only knows a parametrized family of possible waveforms (or approximations thereof). The unknown parameters of these waveforms could be, for example, the frequency and sky position of spinning neutron stars or the masses and spins of inspiralling compact binary systems. Parameter spaces of such wide-parameter searches typically have between one and four dimensions, depending on computational constraints and the amount of astrophysical information available to constrain the search space a priori. In the case of GWs from general binary systems, however, the number of dimensions of the parameter space could be as large as 17 .

Obviously, one can only search a finite subset of points in this parameter space, and this subset constitutes the 'template bank'. The templates must cover the parameter space, i.e., they must be placed densely enough that no signal in this space can lose more than a certain fraction 
of its power (called mismatch) at the closest template. However, coherently correlating the data with every template is computationally expensive and increases the expected number of statistical false-alarm candidates. An optimal template bank therefore consists of the smallest possible number of templates that still guarantees that the worst-case mismatch does not exceed a given limit.

It was realized early on that a geometric approach is very useful to construct template banks, in particular the introduction of a parameter-space metric [3, 11] based on the mismatch. This provides a natural measure of distance in parameter space and allows one to 'correctly' place templates, in the sense that the maximal mismatch is not exceeded. Less attention, however, was devoted to the problem of optimally placing templates once the metric is known. Early works have sometimes used a hyper-cubic template grid for illustrative purposes [11] or the problem was incorrectly referred to as a 'sphere packing problem' $[5,12]$. We see in the following that constructing an optimal template bank is an instance of the sphere covering problem, which is somewhat 'dual' to the sphere packing problem. The full solution to the sphere covering problem in Euclidean space is only known in $n=2$ dimensions, partial solutions (restricted to lattices) are known in $n \leqslant 5$ dimensions, while an optimal solution for higher dimensions is unknown (cf $[8,14])$. The main motivation of the present work is to develop a general method for constructing efficient template banks in dimensions $n \lesssim 17$ by using the known results about Euclidean sphere covering.

Previous related work on template banks includes studies to optimally cover non-flat two-dimensional parameter spaces arising in searches for GWs from inspiralling compact binary systems $[2,4]$. An interesting algorithm for constructing a hexagonal $\left(A_{2}^{*}\right)$ template bank for 2D inspiral searches was described recently in [7]. Various codes exist within the LIGO Scientific Collaboration to generate hyper-cubic lattices (LALCreateFlatMesh() [10]), two-dimensional grids for non-constant metrics (LALCreateTwoDMesh () [10]) and a three-dimensional template bank based on the bcc-lattice (LALInspiralSpinBank () [10]), which is being used in a search for spinning binary inspirals on LIGO Data [1].

\section{Template-based searches and parameter-space metric}

A wide class of searches for GWs can be characterized as template based, in the sense that one searches for signals belonging to a family of waveforms $s(t ; \lambda)$, which depend on a vector of parameters $\{\lambda\}^{i}=\lambda^{i}$. The strain $x(t)$ measured by a detector contains (usually dominating) noise $n(t)$ in addition to possible weak GW signals $s\left(t ; \lambda_{\mathrm{s}}\right)$, i.e., $x(t)=n(t)+s\left(t ; \boldsymbol{\lambda}_{\mathrm{s}}\right)$. One typically constructs a detection statistic, $\mathcal{F}(\lambda ; x)$ say, namely a scalar characterizing the probability of a signal with parameters $\lambda$ being present in the data $x(t)$. Due to the random noise fluctuations $n(t)$ in the data, the detection statistic is a random variable, and generally (assuming $\mathcal{F}$ is unbiased) its expectation value $\overline{\mathcal{F}}\left(\boldsymbol{\lambda} ; \boldsymbol{\lambda}_{\mathrm{s}}\right) \equiv E[\mathcal{F}(\boldsymbol{\lambda} ; x)]$ has a (local) maximum at the location of the signal $\boldsymbol{\lambda}=\boldsymbol{\lambda}_{\mathrm{s}}$, i.e.,

$$
\left.\frac{\partial \overline{\mathcal{F}}\left(\boldsymbol{\lambda} ; \boldsymbol{\lambda}_{\mathrm{s}}\right)}{\partial \boldsymbol{\lambda}}\right|_{\lambda=\lambda_{\mathrm{s}}}=0
$$

Taylor-expanding the expected detection-statistic $\overline{\mathcal{F}}$ in small offsets $\Delta \boldsymbol{\lambda}=\boldsymbol{\lambda}-\boldsymbol{\lambda}_{\mathrm{s}}$ around the signal location $\lambda_{\mathrm{s}}$ therefore reads

$$
\overline{\mathcal{F}}\left(\boldsymbol{\lambda} ; \boldsymbol{\lambda}_{\mathrm{s}}\right)=\overline{\mathcal{F}}\left(\boldsymbol{\lambda}_{\mathrm{s}} ; \boldsymbol{\lambda}_{\mathrm{s}}\right)+\left.\frac{1}{2} \frac{\partial^{2} \overline{\mathcal{F}}\left(\boldsymbol{\lambda} ; \boldsymbol{\lambda}_{\mathrm{s}}\right)}{\partial \lambda^{i} \partial \lambda^{j}}\right|_{\lambda_{\mathrm{s}}} \Delta \lambda^{i} \Delta \lambda^{j}+\mathcal{O}\left(\Delta \lambda^{3}\right),
$$


where the matrix of second derivatives of $\overline{\mathcal{F}}$ is negative definite. Here and in the following we use automatic summation over repeated parameter indices $i, j, \ldots$ We can introduce a mismatch $m$, which characterizes the fractional loss in the expected value of the detection statistic, $\overline{\mathcal{F}}$, at a parameter-space point $\lambda$, with respect to the signal location $\lambda_{\mathrm{s}}$, namely

$$
m\left(\boldsymbol{\lambda} ; \boldsymbol{\lambda}_{\mathrm{s}}\right) \equiv \frac{\overline{\mathcal{F}}\left(\boldsymbol{\lambda}_{\mathrm{s}} ; \boldsymbol{\lambda}_{\mathrm{s}}\right)-\overline{\mathcal{F}}\left(\boldsymbol{\lambda} ; \boldsymbol{\lambda}_{\mathrm{s}}\right)}{\overline{\mathcal{F}}\left(\boldsymbol{\lambda}_{\mathrm{s}} ; \boldsymbol{\lambda}_{\mathrm{s}}\right)}
$$

Using the local expansion (2), we find

$$
m\left(\boldsymbol{\lambda} ; \boldsymbol{\lambda}_{\mathrm{s}}\right)=g_{i j}\left(\boldsymbol{\lambda}_{\mathrm{s}}\right) \Delta \lambda^{i} \Delta \lambda^{j}+\mathcal{O}\left(\Delta \lambda^{3}\right),
$$

where we defined the positive-definite metric tensor $g_{i j} \equiv-\frac{1}{2} \partial_{i} \partial_{j} \overline{\mathcal{F}}$ and $\partial_{i} \equiv \partial / \partial \lambda^{i}$. When searching a parameter space $\mathbb{P}\left(\lambda^{i}, g_{i j}\right)$, we need to compute the detection statistic $\mathcal{F}\left(x ; \lambda_{\xi}\right)$ for a discrete set of templates $\lambda_{\xi} \in \mathbb{P}$. Generally, one can distinguish two different approaches to this problem: one is a random sampling of $\mathbb{P}$ using Markov-chain Monte Carlo (MCMC) algorithms (e.g. see [6,9]), and the other consists of constructing a template bank $\mathbb{T} \equiv\left\{\boldsymbol{\lambda}_{\xi}\right\} \subset \mathbb{P}$ that covers the whole of $\mathbb{P}$, in the sense that no point $\lambda \in \mathbb{P}$ exceeds a given maximal mismatch $m_{\max }$ to its closest template $\boldsymbol{\lambda}_{\xi} \in \mathbb{T}$, i.e.,

$$
\max _{\boldsymbol{\lambda} \in \mathbb{P}} \min _{\boldsymbol{\lambda}_{\xi} \in \mathbb{T}} m\left(\boldsymbol{\lambda} ; \boldsymbol{\lambda}_{\xi}\right) \leqslant m_{\max }
$$

Here, we focus on the construction of optimal template banks, namely those satisfying (5) with the smallest possible number of templates $\lambda_{\xi}$. In the local metric approximation (4), each template $\lambda_{\xi}$ covers a region $B_{\xi}$ of parameter space, namely

$$
B_{\xi}=\left\{\boldsymbol{\lambda} \in \mathbb{P}: g_{i j}\left(\boldsymbol{\lambda}_{\xi}\right) \Delta \lambda^{i} \Delta \lambda^{j} \leqslant m_{\max }, \Delta \boldsymbol{\lambda} \equiv \boldsymbol{\lambda}-\boldsymbol{\lambda}_{\xi}\right\},
$$

which is a sphere of radius $R=\sqrt{m_{\max }}$ in the metric space $\mathbb{P}\left(\lambda^{i}, g_{i j}\right)$. We can therefore reformulate the definition of an optimal template bank as the set of (overlapping) spheres of covering radius $R$ which cover the whole of $\mathbb{P}$ in the sense of (5) with the smallest number of spheres. This is known as the sphere covering problem [8], not to be confused with the somewhat dual sphere packing problem, which seeks to pack the largest number of nonoverlapping 'hard' spheres into a given volume.

\section{The Euclidean sphere covering problem}

In this section, we summarize the current status of the sphere covering problem as far as relevant for the construction of optimal template banks. There has been impressive progress in the study of the covering problem in recent years, e.g. see [8] for a general overview and [14] for a more recent update. Unfortunately, all of these studies are restricted to Euclidean spaces $\mathbb{E}^{n}$, while the metric parameter spaces of GW searches are often curved. In the following, we will therefore make the assumption that $\mathbb{P}\left(\lambda^{i}, g_{i j}\right)$ can be treated as at least approximately flat or can be broken into smaller pieces that can be treated as nearly flat. If the curvature of the metric is too strong, i.e., if the curvature radius is comparable to the covering radius, it will be difficult to make use of the Euclidean covering problem, and a different approach such as a stochastic template bank or an MCMC sampling might be more effective. We further assume that we have found a coordinate system of $\mathbb{P}$ such that the metric components are (approximately) constant, i.e., $g_{i j}(\boldsymbol{\lambda}) \approx$ const $_{i j}$, and for simplicity of notation we assume in this section (without loss of generality) that we have chosen coordinates $x^{i}$ in which the constant-coefficient metric is Cartesian, i.e., $\mathbb{P}=\mathbb{E}^{n}\left(x^{i}, \delta_{i j}\right)$. 


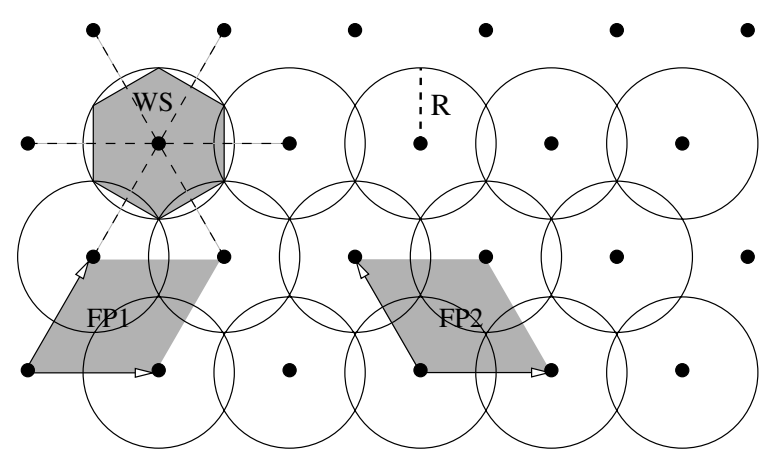

Figure 1. Hexagonal lattice $\left(A_{2}^{*}\right)$ illustrating a two-dimensional lattice covering. The shaded areas are different choices of fundamental regions for the lattice. FP1 and FP2 are fundamental polytopes (9) associated with different choices of lattice basis, WS is the Wigner-Seitz cell (11) and $R$ is the covering radius.

A covering can consist of any arrangement of covering spheres, but currently all the best coverings known are lattices, and we therefore restrict the following discussion to lattice coverings.

\subsection{Basics on lattices}

An $n$-dimensional lattice $\Lambda$ can be defined as a discrete set of points $\boldsymbol{\nu}_{\xi}$ (forming an additive group) generated by

$$
\boldsymbol{\nu}_{\xi}=\xi^{i} \boldsymbol{l}_{(i)}, \quad \text { with } \quad \xi^{i} \in \mathbb{Z},
$$

with summation over $i=1, \ldots, n$, and where $\left\{\boldsymbol{l}_{(i)}\right\}_{i=1}^{n}$ is a basis of the lattice. Note that it is sometimes convenient to express the $n$ basis vectors in a higher-dimensional Euclidean space, i.e., generally we can have $\boldsymbol{l}_{(i)} \in \mathbb{E}^{m}$ with $m \geqslant n$. When writing $\mathbb{E}^{n}$ in the following we refer to the subspace of $\mathbb{E}^{m}$ containing the $n$-dimensional lattice $\Lambda$. The $m \times n$ matrix $M^{a}{ }_{i} \equiv l_{(i)}^{a}$ is called a generator matrix of the lattice, with the columns of $M$ holding the $m$ components of the $n$ lattice basis vectors, so we can also write the lattice $\Lambda$ as

$$
\Lambda=\left\{\nu_{\xi}: \nu_{\xi}=M \xi, \xi \in \mathbb{Z}^{n}\right\} .
$$

The $n \times n$ matrix $A \equiv M^{\mathrm{T}} M$ is called the Gram matrix (where T denotes the transpose), which is symmetric and positive definite, and $A_{i j}=l_{(i)} \cdot l_{(j)}=\delta_{a b} l_{(i)}^{a} l_{(j)}^{b}$, i.e., its coefficients are the mutual scalar products of lattice basis vectors. Each choice of lattice basis $\left\{\boldsymbol{l}_{(i)}\right\}$ defines a corresponding fundamental parallelotope (FP), namely

$$
\operatorname{FP}\left(\left\{\boldsymbol{l}_{(i)}\right\}\right) \equiv\left\{\boldsymbol{x} \in \mathbb{E}^{n}: \boldsymbol{x}=\theta^{i} \boldsymbol{l}_{(i)}, 0 \leqslant \theta^{i}<1\right\},
$$

which is illustrated in figure 1. The FP is an example of a fundamental region for the lattice, i.e., a building block containing exactly one lattice point, which fills the whole space $\mathbb{E}^{n}$ when repeated. There are many different choices of basis and fundamental regions for the same lattice $\Lambda$, but they all have the same $\operatorname{volume} \operatorname{vol}(\Lambda)$, given by

$$
\operatorname{vol}(\Lambda)=\sqrt{\operatorname{det} A}
$$

and in the case where $M$ is a square matrix we also have $\operatorname{vol}(\Lambda)=\operatorname{det} M$. One special choice of fundamental region is the nearest-neighbor region, often referred to as Dirichlet-Voronoi cell by mathematicians, and more commonly known as Wigner-Seitz cell or Brillouin zone by physicists, which is defined as

$$
\mathrm{WS}(\Lambda) \equiv\left\{\boldsymbol{x} \in \mathbb{E}^{n}:\left\|\boldsymbol{x}-\boldsymbol{\nu}_{0}\right\| \leqslant\left\|\boldsymbol{x}-\boldsymbol{\nu}_{\xi}\right\|, \text { for all } \boldsymbol{\nu}_{\xi} \in \Lambda\right\},
$$


where $\|\boldsymbol{x}\|=\sqrt{\boldsymbol{x} \cdot \boldsymbol{x}}$ is the standard Euclidean norm in $\mathbb{E}^{n}$. The vertices of the Wigner-Seitz cell are by construction local maxima of the distance function of points in $\mathbb{E}^{n}$ from the nearest grid point. The maximum distance of any point in $\mathbb{E}^{n}$ to the nearest point of the lattice is called the covering radius $R$, which corresponds to the circumradius of WS, as seen in figure 1.

Two lattices $\Lambda_{1}$ and $\Lambda_{2}$ with generator matrices $M_{1}$ and $M_{2}$ are equivalent if they can be transformed into one another by a rotation, reflection and change of scale, namely if the generator matrices satisfy

$$
M_{2}=c B M_{1} U
$$

where $c \in \mathbb{R}$ is a scale factor, $U$ is integer-valued $\operatorname{det} U= \pm 1$, which accounts for different choices of basis vectors, and $B$ is a real orthogonal matrix, i.e., $B^{\mathrm{T}} B=\mathbb{I}$. The associated Gram matrices are therefore related by

$$
A_{2}=c^{2} U^{\mathrm{T}} A_{1} U,
$$

and the fundamental volumes (10) of the two lattices are

$$
\operatorname{vol}\left(\Lambda_{2}\right)=c^{n} \operatorname{vol}\left(\Lambda_{1}\right) .
$$

Let us consider as an example the two-dimensional hexagonal lattice, illustrated in figure 1 . An obvious generator matrix is

$$
M_{1}=\left(\begin{array}{cc}
1 & 1 / 2 \\
0 & \sqrt{3} / 2
\end{array}\right)
$$

corresponding to FP1 in figure 1. However, sometimes it is more convenient to work with a generator matrix of the form

$$
M_{2}=\left(\begin{array}{cc}
1 & 0 \\
-1 & 1 \\
0 & -1
\end{array}\right)
$$

which has simpler coefficients, but uses a three-dimensional representation of the twodimensional lattice with all lattice points lying in the plane $x+y+z=0$. One can verify that these two representations are equivalent in the sense of (12), namely with

$$
c=\sqrt{2}, \quad U=\left(\begin{array}{cc}
1 & 0 \\
0 & -1
\end{array}\right), \quad B=\left(\begin{array}{cc}
1 / \sqrt{2} & -1 / \sqrt{6} \\
-1 / \sqrt{2} & -1 / \sqrt{6} \\
0 & \sqrt{2 / 3}
\end{array}\right) .
$$

Such a higher-dimensional representation of the generator matrix will be useful later for the description of the $n$-dimensional $A_{n}^{*}$ lattice.

\subsection{Known results on optimal sphere covering}

The efficiency of a sphere covering can be characterized by its thickness $\Theta$ (sometimes also referred to as the covering density), which measures the fractional amount of overlap between the covering spheres or, equivalently, the average number of spheres covering any point in $\mathbb{E}^{n}$. This can be expressed as the ratio of the volume of one covering sphere to the volume of the fundamental region of the lattice, i.e.,

$$
\Theta \equiv \frac{V_{n} R^{n}}{\operatorname{vol}(\Lambda)} \geqslant 1
$$


where $R$ is the covering radius and $V_{n}$ is the volume of the unit sphere in $n$ dimensions, namely $V_{n}=\pi^{n / 2} / \Gamma(n / 2+1)$. We also use the normalized thickness or center density $\theta$ defined as

$$
\theta \equiv \frac{\Theta}{V_{n}}
$$

which corresponds to the number of centers (i.e., templates) per unit volume in the case of $R=1$. Note that under a lattice transformation (12), the covering radius $R$ obviously scales as $R_{2}=c R_{1}$, and we therefore see from (14) that the thickness (18) and (19) is an invariant property of a lattice, i.e., $\theta_{2}=\theta_{1}$. The covering problem consists of finding the covering with the lowest center density $\theta$.

Kershner showed in 1939 (see [8]) that in $n=2$ dimensions the most economical arrangement of circles covering the plane is the hexagonal lattice, which is equivalent to an $A_{2}^{*}$ lattice. In dimensions $n=3,4,5$ only the best lattice covering is known, and is given by $A_{n}^{*}$ in all three cases. In three dimensions, $A_{3}^{*}$ is also known as the body-centered-cubic (bcc) lattice. Note that the best packing in $n=2$ is also achieved by the hexagonal lattice, but for $n=3$ the face-centered cubic (fcc) lattice provides a denser packing than bcc. In higher dimensions the best lattice coverings are currently still unknown, but the best coverings known can be found in table 2 of [14], and [15] provides an up-to-date online version. As will become clearer in the following, the $A_{n}^{*}$ lattice, while no longer the 'record holder' for most dimensions $5<n \leqslant 17$, is still close to the best currently known covering in all cases. In the following, we will therefore mostly focus on the $A_{n}^{*}$ covering. The $A_{n}^{*}$ lattice has a center density of

$$
\theta\left(A_{n}^{*}\right)=\sqrt{n+1}\left\{\frac{n(n+2)}{12(n+1)}\right\}^{n / 2},
$$

while for the hyper-cubic grid $\mathbb{Z}^{n}$ the Wigner-Seitz cell is a unit hypercube, $\operatorname{so} \operatorname{vol}\left(\mathbb{Z}^{n}\right)=1$, and the covering radius $R=\sqrt{n} / 2$ is half the length of the diagonal. Therefore, the center density (19) is found as $\theta\left(\mathbb{Z}^{n}\right)=2^{-n} n^{n / 2}$, which is dramatically worse than $A_{n}^{*}$ in higher dimensions, as can be seen from the thickness ratio

$$
\kappa(n) \equiv \frac{\theta\left(\mathbb{Z}^{n}\right)}{\theta\left(A_{n}^{*}\right)}=\frac{3^{n / 2}}{\sqrt{n+1}}\left(\frac{n+1}{n+2}\right)^{n / 2} \stackrel{n \rightarrow \infty}{\sim} \frac{3^{n / 2}}{\sqrt{n e}} .
$$

There is a theoretical lower limit on the thickness of any covering, the Coxeter-Few-Rogers (CFR) bound $\tau_{n}$ (see [8]), i.e., $\theta_{n} \geqslant \tau_{n} / V_{n}$, where asymptotically $\tau_{n} \sim n /(e \sqrt{e})$ for $n \rightarrow \infty$. Figure 2 shows the normalized thickness $\theta$ as a function of dimension $n$ for the $A_{n}^{*}$ and hyper-cubic $\mathbb{Z}^{n}$ lattices, as well as the CFR bound and the best covering known. In table 1 , we see that in dimensions $n>5$, where $A_{n}^{*}$ has been superseded as the best covering [15], the relative improvement $\gamma(n) \equiv \theta$ (best) $/ \theta\left(A_{n}^{*}\right)$ in thickness is typically quite small. In particular, for $n \leqslant 16$ the improvement $\gamma(n)$ is typically less than $18 \%$, while the advantage $\kappa(n)$ of $A_{n}^{*}$ compared to the hyper-cubic grid $\mathbb{Z}^{n}$ grows large very rapidly, as seen in table 1 and figure 2 . For practical simplicity we therefore propose to use $A_{n}^{*}$ as the covering lattice of choice.

\section{Lattice covering of template spaces}

\subsection{Template counting}

The template spaces $\mathbb{P}\left(\lambda^{i}, g_{i j}\right)$ with constant-coefficient metrics $g_{i j}$ only differ from the Cartesian case of the previous section by a simple coordinate transformation. An infinitesimal 


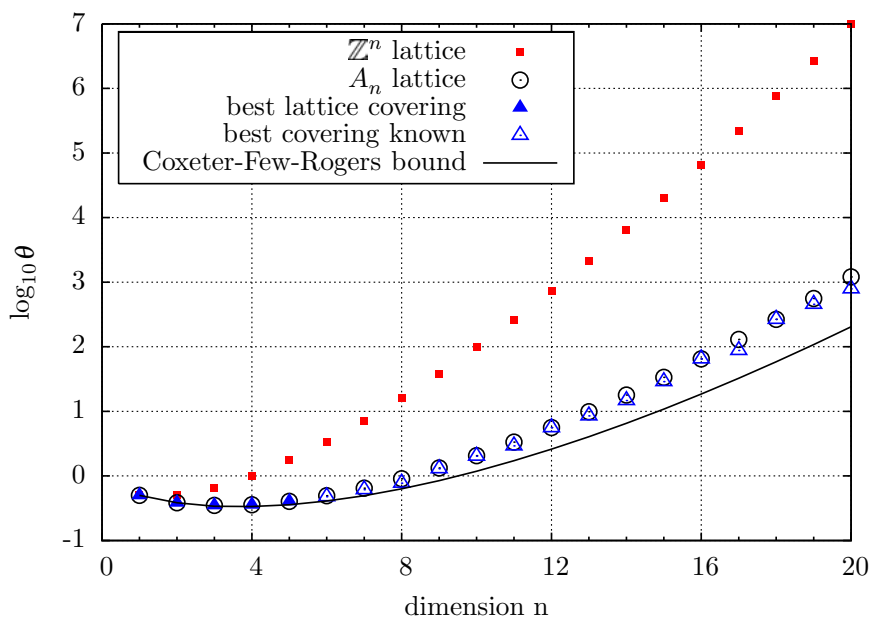

Figure 2. Normalized covering thickness $\theta$ as a function of dimension $n$, for the hyper-cubic lattice $\left(\mathbb{Z}^{n}\right)$, the $A_{n}^{*}$ lattice, the theoretical lower bound (CFR) and the best lattice coverings known.

Table 1. Thickness ratio $\kappa(n)=\theta\left(\mathbb{Z}^{n}\right) / A_{n}^{*}$ and $\gamma(n)=\theta$ (best) $/ \theta\left(A_{n}^{*}\right)$ in dimensions $n \leqslant 17$.

\begin{tabular}{lllllllll}
\hline$n$ & 2 & 3 & 4 & 5 & 6 & 7 & 8 & 9 \\
\hline$\kappa(n)$ & 1.3 & 1.9 & 2.8 & 4.3 & 6.8 & 10.9 & 17.7 & 28.9 \\
$\gamma(n)$ & 1.0 & 1.0 & 1.0 & 1.0 & 0.97 & 0.95 & 0.86 & 0.97 \\
\hline$n$ & 10 & 11 & 12 & 13 & 14 & 15 & 16 & 17 \\
\hline$\kappa(n)$ & 47.4 & 78.2 & 130 & 216 & 359 & 601 & 1007 & 1692 \\
$\gamma(n)$ & 0.98 & 0.88 & 0.99 & 0.86 & 0.82 & 0.86 & 1.0 & 0.68 \\
\hline
\end{tabular}

parameter-space region $\mathrm{d}^{n} \lambda$ has a volume $\mathrm{d} V$ measured by the metric, namely $\mathrm{d} V=\sqrt{g} \mathrm{~d}^{n} \lambda$, where $g \equiv \operatorname{det} g_{i j}$. The volume $V$ of a finite region of parameter space is therefore

$$
V=\int_{\mathbb{P}} \mathrm{d} V=\sqrt{g} \int_{\mathbb{P}} \mathrm{d}^{n} \lambda
$$

where we used the fact that $g_{i j}$ is a constant-coefficient metric. The number of templates $\mathrm{d} N_{p}$ in $\mathrm{d} V$ is given by the inverse lattice volume, i.e.,

$$
\mathrm{d} N_{p}=\frac{\mathrm{d} V}{\operatorname{vol}(\Lambda)}
$$

Using the relation $R=\sqrt{m_{\max }}$ together with (18), (19), we find

$$
\mathrm{d} N_{p}=\theta m_{\max }^{-n / 2} \mathrm{~d} V \quad \Longrightarrow \quad N_{p}=\theta m_{\max }^{-n / 2} \sqrt{g} \int_{\mathbb{P}} \mathrm{d}^{n} \lambda,
$$

which generalizes template counting $[5,11,12]$ to arbitrary lattices.

\subsection{Practical implementation of lattice covering}

In this section, we present a practical algorithm for generating lattices covering of given maximal mismatch $m_{\max }$. The approach described here works for any lattice generator $M$, but 
in practice (cf section 3.2) we will be most interested in the $A_{n}^{*}$ lattice. The generator for $A_{n}^{*}$ can be expressed (cf [8]) as an $(n+1) \times n$ matrix ,

$$
M^{a}{ }_{j}\left(A_{n}^{*}\right)=\left(\begin{array}{ccccc}
1 & 1 & \ldots & 1 & \frac{-n}{n+1} \\
-1 & 0 & \ldots & 0 & \frac{1}{n+1} \\
0 & -1 & \ldots & 0 & \frac{1}{n+1} \\
\vdots & \vdots & \vdots & \vdots & \vdots \\
0 & 0 & \ldots & -1 & \frac{1}{n+1} \\
0 & 0 & \ldots & 0 & \frac{1}{n+1}
\end{array}\right),
$$

where the columns of $M$ hold the $n$ lattice basis vectors $\boldsymbol{l}_{(j)}$ expressed in $\mathbb{E}^{n+1}$, i.e., $M^{a}{ }_{j}=l_{(j)}^{a}$, with index conventions $i, j=1, \ldots, n$ and $a, b=1, \ldots, n+1$. The volume of the fundamental region and the covering radius for this generator are

$$
\operatorname{vol}\left(A_{n}^{*}\right)=\frac{1}{\sqrt{n+1}} \quad \text { and } \quad R\left(A_{n}^{*}\right)=\sqrt{\frac{n(n+2)}{12(n+1)}},
$$

which yields the (normalized) thickness $\theta\left(A_{n}^{*}\right)$ given in (20). In order to generate such a lattice in a parameter space $\mathbb{P}\left(\lambda^{i}, g_{i j}\right)$, we need to express the generator $M^{a}{ }_{j}$ in the $\lambda^{i}$ coordinates, resulting in $\widetilde{M}_{j}^{i}$, say, such that the lattice of templates $\boldsymbol{\lambda}_{\xi}$ is generated by

$$
\lambda_{\xi}^{i}=\widetilde{M}_{j}^{i} \xi^{j}, \quad \text { with } \quad \xi \in \mathbb{Z}^{n} .
$$

This coordinate transformation can be achieved in several steps:

(1) Reduce the $(n+1) \times n$ matrix $M^{a}{ }_{j}$ to a full rank generator, $\widehat{M}_{j}^{i}$ say, by expressing the lattice basis vectors in a Euclidean basis spanning the $n$-dimensional subspace $\mathbb{E}^{n}$ of the lattice: a simple Gram-Schmidt procedure with respect to the Cartesian metric $\delta_{a b}$ is used on $\left\{l_{(j)}^{a}\right\}$ to generate an orthonormal basis $\left\{e_{(j)}^{a}\right\}$ satisfying

$$
\delta_{a b} e_{(i)}^{a} e_{(j)}^{b}=\delta_{i j} .
$$

The full-rank generator $\widehat{M}_{j}^{i}$ is obtained from the components of the lattice vectors $\left\{l_{(i)}^{a}\right\}$ in this orthonormal basis, namely

$$
\widehat{M}_{j}^{i}=\widehat{l}_{(j)}^{i}=l_{(j)}^{a} e_{(i)}^{b} \delta_{a b}=e_{(i) a} M^{a}{ }_{j} .
$$

(2) Translate the full-rank generator $\widehat{M}_{j}^{i}$ from Cartesian coordinates into the coordinate system $\lambda^{i}$ with metric $g_{i j}$. For this we use another Gram-Schmidt orthonormalization with respect to the metric $g_{i j}$, with the lattice vectors $\left\{\hat{l}_{(i)}^{j}\right\}$ as input to find an orthonormal basis $\left\{d_{(i)}^{j}\right\}$ satisfying

$$
g_{i j} d_{(l)}^{i} d_{(k)}^{j}=\delta_{l k} .
$$

This representation of an orthonormal basis in coordinates $\lambda^{i}$ allows us to express the lattice vectors in these coordinates as

$$
\widetilde{l}_{(j)}^{i}=\widehat{l}_{(j)}^{k} d_{(k)}^{i}=d_{(k)}^{i} \widehat{M}_{j}^{k} \text {. }
$$

(3) Scale the generator to the desired covering radius $R=\sqrt{m_{\max }}$, and with (26) we find

$$
\tilde{M}_{j}^{i}=\sqrt{m_{\max }} \sqrt{\frac{12(n+1)}{n(n+2)}} \widetilde{l}_{(j)}^{i},
$$

which is a generator (27) for an $A_{n}^{*}$ template lattice with maximal mismatch $m_{\max }$.

This algorithm has been implemented in XLALFindCoveringGenerator () in LAL [10], and some tests of this code are presented in the following section. 

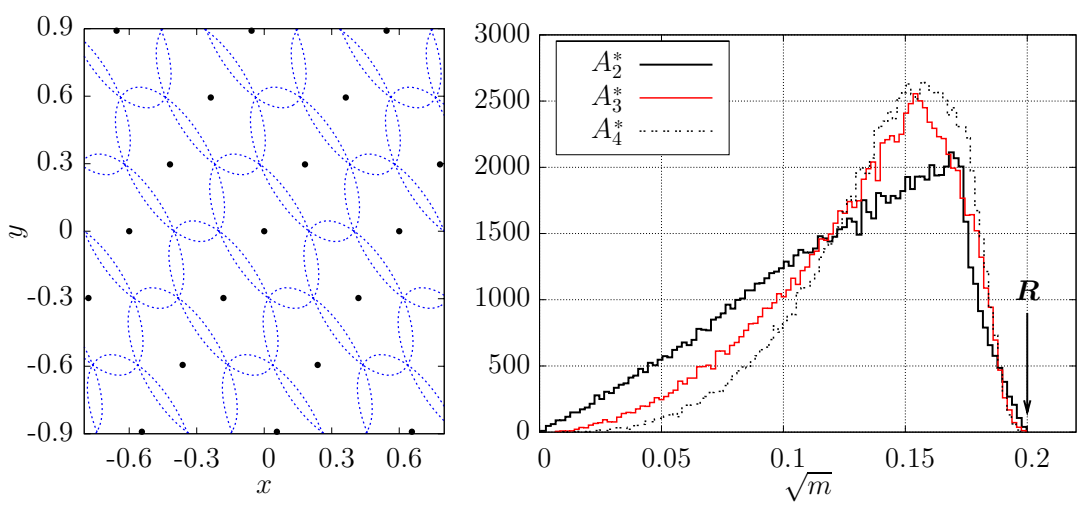

Figure 3. Left panel: hexagonal $\left(A_{2}^{*}\right)$ lattice covering in coordinates $\{x, y\}$ with metric $g_{i j}=[1,0.4 ; 0.4,0.5]$. Right panel: histogram of measured distances $\sqrt{m}$ in a Monte Carlo sampling of 100000 points from an $A_{n}^{*}$ covering in $n=2,3,4$ dimensions, using non-Cartesian metrics $g_{i j}$. The nominal covering radius in all three cases was $R=\sqrt{m_{\max }}=0.2$.

\subsection{Tests of the implementation}

In order to illustrate and test the implementation of this algorithm, we generate an $A_{n}^{*}$ lattice in dimensions $n=2,3,4$, respectively, with a maximal mismatch of $m_{\max }=0.04$, i.e., a covering radius of $R=0.2$. For generality we use a non-Cartesian metric $g_{i j} \neq \delta_{i j}$, as illustrated in the left panel of figure 3 . We picked 100000 points $\lambda \in \mathbb{P}\left(\lambda^{i}, g_{i j}\right)$ at random and computed their mismatch $m$ (using the metric) to the nearest template $\boldsymbol{\lambda}_{\xi}$, which is a way of measuring the maximal mismatch of a template bank. The distribution of measured mismatch distances $\sqrt{m}$ is plotted on the right-hand panel of figure 3, and we see that the mismatches are bounded by $\sqrt{m_{\max }}=0.2$, satisfying (5). We can also measure the (normalized) thickness $\theta$ of the template bank, namely from the number of templates $N_{p}$ in the covered parameter space $\Delta \lambda^{n}$, we find using (24)

$$
\theta=\frac{R^{n}}{\sqrt{g}} \frac{N_{p}}{\Delta \lambda^{n}} .
$$

These measured values of the thickness are found to agree to within $0.2 \%$ with the theoretical values (20) in all three cases $n=2,3,4$. The generated template banks in this example have $N_{p} \sim \mathcal{O}\left(10^{4}\right)$ templates, and the error can most likely be attributed to boundary effects.

\section{Discussion}

Possible applications of this algorithm for GW searches can be found in template-based searches, such as for inspiralling compact binary systems and for 'continuous waves', which in ground-based detectors refers mostly to signals from spinning neutron stars, and in the case of LISA includes white dwarf binaries, supermassive black hole binaries and extrememass ratio inspirals. The benefit of using this approach depends sensitively on the number of parameter-space dimensions, but can be estimated from table 1 at least in comparison to hypercubic grids.

However, the applicability of the lattice covering algorithm presented here is restricted to explicitly flat parameter spaces, which limits its usefulness to cases where we can find a coordinate system in which the parameter-space metric is (at least) approximately constant. The orbital metric approximation [13] for continuous GWs can be shown to be flat (work in 
progress) and would therefore be a natural case where this lattice covering could be used to greatest effect. One difficulty in this case, however, stems from the fact that the corresponding metric is found to be highly ill-conditioned, which results in the lattice-construction algorithm to fail due to numerical problems. One therefore needs to analytically 'factor out' this near degeneracy of the metric before this lattice-covering procedure can be safely applied. More work is also required to deal with non-trivial parameter-space boundaries, which complicates the $n$-dimensional filling algorithm.

\section{Acknowledgments}

I am grateful for discussions with Bruce Allen, Andrzej Królak and Curt Cutler. I further thank Achill Schürmann for reading the manuscript and providing helpful comments. This work was supported by the Max-Planck-Society (AEI-2007-016). This paper has been assigned LIGO Document Number LIGO-P070030-02-Z.

\section{References}

[1] Abbott B et al (LIGO Scientific Collaboration) in preparation

[2] Babak S, Balasubramanian R, Churches D, Cokelaer T and Sathyaprakash B S 2006 Class. Quantum Grav. 235477

[3] Balasubramanian R, Sathyaprakash B S and Dhurandhar S V 1996 Phys. Rev. D. 533033

[4] Beauville F, Buskulic D, Flaminio R, Gouaty R, Grosjean D, Marion F, Mours B, Tournefier E, Verkindt D and Yvert M 2005 Class. Quantum Grav. 224285

[5] Brady P R, Creighton T, Cutler C and Schutz B F 1998 Phys. Rev. D. 572101

[6] Christensen N, Dupuis R J, Woan G and Meyer R 2004 Phys. Rev. D. 70022001

[7] Cokelaer T 2007 Preprint 0706.4437

[8] Conway J H and Sloane N J A 1999 Sphere Packings, Lattices and Groups (Berlin: Springer)

[9] Cornish N J and Crowder J 2005 Phys. Rev. D. 72043005

[10] LIGO Scientific Collaboration, LAL/LALApps: FreeSoftware (GPL) tools for data-analysis http://www.lscgroup.phys.uwm.edu/daswg

[11] Owen B J 1996 Phys. Rev. D. 536749

[12] Owen B J and Sathyaprakash B S 1999 Phys. Rev. D. 60022002

[13] Prix R 2007 Phys. Rev. D. 75023004

[14] Schürmann A and Vallentin F 2006 Discrete and Comput. Geometry 3573

[15] Schürmann A and Vallentin F 2007 http://www.math.uni-magdeburg.de/lattice_geometry (cited 22 March 2007) 